\title{
VARIAÇÃO DO ÍNDICE DE ÁREA FOLIAR EM CLONES DE EUCALIPTO AO LONGO DE SEU CICLO DE CRESCIMENTO ${ }^{1}$
}

\begin{abstract}
Alexandre Cândido Xavier² ${ }^{2}$ João Vianei Soares ${ }^{3}$ e Auro Campi de Almeida ${ }^{4}$
RESUMO - O índice de área foliar (IAF) é uma das principais variáveis biofísicas de um dossel florestal, estando diretamente relacionado com a sua evapotranspiração e sua produtividade. Este trabalho apresenta um levantamento da variabilidade temporal do IAF em cinco diferentes clones (designados para efeito deste trabalho como MG1, MG2, MG3, MG4 e MG5) de plantações de eucalipto (híbridos de Eucalyptus grandis), na regional de produção de Aracruz-ES, Brasil. Utilizou-se o equipamento LAI-2000 para medir o IAF em 98 talhões, com didade variando de 12 a 84 meses. o IAF variou de 1,7 a 4,3. Houve correlação negativa significativa entre IAF e idade para os clones MG1, MG2, e MG3. Para os clones MG4 e MG5 foi constatado que não houve relação significativa entre IAF e idade, o que evidencia que sejam adotados valores médios de IAF destes clones como descritores estruturais do dossel para fins de modelagem ou outros (2,57 e 3,04, respectivamente).
\end{abstract}

Palavras-chave: LAI-2000, plantações de eucalipto, índice de área foliar e clones.

\section{LEAF AREA INDEX CHANGES WITH AGE AMONG EUCALYPT CLONAL PLANTATIONS}

\begin{abstract}
The leaf area index (LAI) is one of the key variables for canopy structure description, as it is directly related with evapotranspiration and primary production. This paper presents LAI variation with age for five different genetic materials (MG1, MG2, MG3, MG4 and MG5) of eucalypt plantations (Eucalyptus grandis hybrids), in the eastern Atlantic coast of Brazil. The LAI-2000 Plant Canopy Analyzer was used to estimate LAI in 98 stands, with ages ranging from 12 to 84 months. LAI values ranged from 1.7 to 4.3. A negative correlation was observed between LAI and age for genotypes MG1, MG2 and MG3, whereas no significant correlation was detected for clones MG4 and MG5. For applications such as process-based models used to estimate NPP and evapotranspiration for MG4 and MG5, LAI average values of 2.57 and 3.04, respectively, must be used.
\end{abstract}

Key words: $\quad$ LAI-2000, eucalypt plantation, leaf area index, clones.

\section{INTRODUÇÃO}

As variáveis biofísicas são importantes descritoras de florestas nativas ou implantadas. Por exemplo, a altura e o diâmetro à altura do peito das árvores (DAP) são frequientemente utilizados em equações alométricas para o cálculo de volume de madeira. Outra variável biofísica importante é o índice de área foliar (IAF), definido por Watson (1947) como a área foliar integrada do dossel por unidade de superfície projetada no solo $\left(\mathrm{m}^{2} / \mathrm{m}^{2}\right)$. O IAF é computado ao considerar a superfície de apenas uma das faces das folhas.

1 Recebido para publicação em 8.10.2001.

Aceito para publicação em 20.6.2002.

Parte da dissertação de Mestrado, apresentada pelo primeiro autor (Bolsista da CAPES) no Instituto Nacional de Pesquisas Espaciais (INPE), São José dos Campos.

2 Universidade de São Paulo/ESALQ, Departamento de Engenharia Rural, Caixa Postal 09, 13418-900 Piracicaba-SP, <acxavier@carpa.ciagri.usp.br>. ${ }^{3}$ Instituto Nacional de Pesquisas Espaciais, Av. dos Astronautas, 1758, 12227-010 São José dos Campos-SP, <vianei@ltid.inpe.br>. ${ }^{4}$ Aracruz Celulose S.A. Rodovia Aracruz/Barra do Riacho, Caixa Postal 331011, 29192-000 Aracruz-ES, <aca@aracruz.com.br>. 
O IAF está diretamente relacionado com a produtividade e a evapotranspiração de ecossistemas florestais (Lang \& Mcmurtrie, 1992). Para estimar a produtividade e a evapotranspiração, pesquisadores têm desenvolvido modelos de interface floresta-solo-atmosfera (Running \& Coughlan, 1988), nos quais o IAF é a principal variável descritora do dossel vegetal. Soares et al. (1997) e Soares \& Almeida (2001) adaptaram o método de PenmanMonteith para estimar a transpiração e o balanço hídrico para plantações de eucalipto, em que o IAF é utilizado para estimar a condutância do dossel, parâmetro-chave no modelo, a partir de valores amostrados para folhas individuais. Ainda, para Soares et al. (1997) e Soares \& Almeida (2001), a evapotranspiração do dossel, quando integrada em um dado período de tempo, pode ser utilizada para expressar o potencial de produtividade da cultura em determinado local e período.

A definição detalhada da estrutura do dossel requer a inclusão de outros atributos, como tamanho, tipo, orientação e distribuição posicional das várias partes da planta, como folhas, galhos, troncos, flores e frutos. Segundo Norman \& Campbell (1989), as descrições da estrutura de dosséis são essenciais para o entendimento dos processos de troca entre a planta e a atmosfera, como energia e momento, mas também podem revelar estratégias da planta no seu processo evolutivo, como adaptações físico-químicas. Infelizmente, as descrições quantitativas das características geométricas do dossel, das plantas ou dos organismos individuais são complexas, pois os dosséis variam tanto espacial quanto temporalmente (sazonalmente e idade). Desta forma, a utilização de IAF como um descritor integrado da estrutura do dossel parece ser um bom compromisso, sobretudo se o objeto de análise são as partições de energia e de momento entre o dossel e o solo.

Do ponto de vista de plantações, por exemplo de eucalipto, é importante quantificar o IAF, assim como a sua variação ao longo de seu ciclo produtivo, pois a partir desta quantificação torna-se possível sua utilização como variável de entrada em modelos hidrológicos e de crescimento. Computar o uso de água de plantações de eucalipto é fundamental tanto na avaliação de seus impactos ambientais quanto na aferição de sua sustentabilidade. Os modelos de crescimento permitem, por outro lado, simular o efeito de variações climáticas e da fertilidade do solo sobre o potencial produtivo dessas plantações.

Os métodos de medição e estimação do IAF podem ser classificados como diretos e indiretos. Daughtry
(1990) descreveu diferentes métodos diretos de medição do IAF, entre os quais se destacam: a) método de delineamento da folha; b) método com base na combinação de formas e tamanhos- padrão; c) método de cálculo com base em medidas lineares; d) método com base na relação entre área e massa foliar; e e) método da planimetria óptica. Os métodos de medição indireta do IAF são, usualmente, baseados na medição da atenuação de radiação pelo dossel, a partir da teoria de transferência de radiação. Welles (1990) e Welles \& Cohen (1996) descreveram uma série de instrumentos e métodos para realizar estas medições, entre os quais estão as fotografias hemisféricas, o sensor quântico de linha, DEMON, e o LAI-2000. Em geral, os métodos de medida direta apresentam maior precisão, mas são mais laboriosos que os indiretos.

O equipamento LAI-2000 (LI-COR, 1992) vem sendo amplamente utilizado em áreas florestais, devido à sua praticidade para estimar o IAF. Entre os autores que utilizaram esta técnica estão Vertessy et al. (1995), Chen \& Cihlar (1996), Hingston et al. (1998), Ares \& Fownes (2000), Barclay et al. (2000) e Linhares et al. (2000).

O LAI-2000 utiliza um sensor de luz que mede, simultaneamente, a radiação difusa em cinco bandas angulares distintas $\left(0-13^{\circ}, 16-28^{\circ}, 32-43^{\circ}, 47-58^{0}\right.$ e $61-$ $74^{\circ}$ ). O sensor é constituído de cinco fotodiodos, cujas superfícies ativas estão arranjadas em anéis concêntricos. A imagem do dossel, em forma quase hemisférica, é projetada dentro desses anéis, o que permite, para cada intervalo angular, a medição da radiação na banda e no ângulo zenital conhecido. Um filtro óptico restringe a transmissão radiativa para comprimentos de onda menores que $490 \mathrm{~nm}$ (LI-COR, 1992). Alguns estudos mostram que o equipamento LAI-2000, quando utilizado em áreas florestais, subestima o IAF quando este está acima de 2,0, sendo indicada a calibração do equipamento para as condições de uso local com base em amostras destrutivas (Chen et al., 1991; Battaglia et al., 1998). A violação de algumas preposições para utilização do equipamento LAI-2000 seria uma das causas dessa subestimativa, como a distribuição não-aleatória das folhas no dossel (Chen et al., 1991). Este trabalho é a primeira fase para o entendimento do curso da variável IAF em condições de campo, ao longo da idade no ciclo de crescimento de plantações de eucalipto.

O objetivo central deste trabalho foi avaliar como o índice de área foliar varia ao longo do ciclo de 
crescimento de plantações de eucalipto, em cinco clones de híbridos de Eucalyptus grandis W. Hill ex Maiden.

\section{MATERIAL E MÉTODOS}

\subsection{Campanha de Campo}

O trabalho de campo foi conduzido no norte do Estado do Espírito Santo, na área florestal pertencente à Aracruz Celulose S.A, localizada entre as latitudes $19^{\circ} 39^{\prime} 00^{\prime \prime}$ e $20^{\circ} 00^{\prime} 05^{\prime \prime}$ sul e longitudes $43^{\circ} 43^{\prime} 30^{\prime \prime}$ e $44^{\circ} 00^{\prime} 00^{\prime \prime}$ oeste. A coleta de dados ocorreu no período de abril a junho de 1997. Primeiramente, foram escolhidos os clones representativos para medição de IAF (Quadro 1), seguindo o critério de importância relativa em termos de área plantada. Procurou-se, também, escolher os clones que possuíssem maior amplitude de variação em classes de idade, no intuito de verificar o tipo de relação do IAF com a idade. Uma vez escolhidos os clones, gerou-se um mapa temático em um sistema de informações geográficas (SIG), contendo a identificação do clone, a classe de idade e o tipo de manejo (reforma ou rebrota). A partir deste mapa temático foram selecionados 98 talhões para coleta dos dados IAF, nas áreas de plantio de eucalipto. $\mathrm{O}$ espaçamento entre árvores das plantações de eucalipto da regional de produção estudada é de $3 \times 3 \mathrm{~m}$.

A estimativa do IAF, no campo, foi realizada por duas equipes, cada uma dispondo de um par de equipamentos LAI-2000. Para cada equipe, um medidor coletava dados fora do dossel e o outro, sob o dossel.

As medidas de campo foram realizadas, preferencialmente, sob luz difusa, no final da tarde. Os medidores foram instalados e verificados, sistematicamente, antes do início da coleta. O equipamento foi posicionado sempre na direção oposta ao sol (apontado para leste), para

Quadro 1 - Número de talhões por clone Table 1 - Number of blocks planted by genetic material

\begin{tabular}{|c|c|}
\hline Clones & Número de Talhões \\
\hline MG1 & 22 \\
MG2 & 24 \\
MG3 & 12 \\
MG4 & 14 \\
MG5 & 26 \\
\hline
\end{tabular}

garantir condições de iluminação similares. Testes específicos para verificar a homogeneidade nos horários de medição e na direção de alinhamento do equipamento, no momento da tomada de dados, mostraram resultados idênticos de IAF, quando comparados às medidas realizadas sob condições controladas de luz difusa (Xavier, 1998). Em cada talhão foram realizadas 25 leituras, sempre que possível distribuídas em cinco ruas, fazendose cinco leituras por rua, em pontos espaçados em aproximadamente $5 \mathrm{~m}$ entre si, para representar o IAF médio do talhão. Para efeito de comparação, foram também gerados números aleatórios de direção e distância para um esquema amostral aleatório, e os resultados de IAF foram idênticos (Xavier, 1998).

Na coleta de dados de IAF, foi estabelecido um banco de dados do qual constavam a localização do talhão, a idade, a descrição do tipo de sub-bosque, o IAF e o desvio-padrão da amostra.

\subsection{Relação Entre o IAF e a Idade}

Para avaliar a variação do IAF nos diferentes clones, foi utilizada a análise de regressão por mínimos quadrados, (SAS Institute Inc., 1990), com a idade como variável independente. Os modelos investigados foram do tipo linear, potencial e polinomial quadrático. Os parâmetros dos modelos foram testadas 1\% (valor-p (nível observado de significância) <0,01). Foi utilizada a análise de resíduo para identificar a violação do modelo. Os resultados são reportados em termos de coeficiente de determinação $\left(\mathrm{R}^{2}\right)$.

\section{RESULTADOS E DISCUSSÃO}

A faixa de variação da idade foi de 15 a 78 meses. Buscou-se, desta forma, cobrir a maior amplitude possível de idades no ciclo produtivo da plantação de eucalipto, que é de 7 anos (84 meses) para o corte.

O IAF médio do total das 98 amostras (Quadro 2) foi de 2,86 $\left(S_{I A F}=0,47\right)$, valor muito próximo de 2,75 , obtido por Kallarackal \& Somen (1997) em plantações de Eucalyptus grandis de 4 anos, e maior que a média encontrada por Hingston et al. (1998), de 2,30 ( $\left.S_{I A F}=0,30\right)$, em plantações de Eucalyptus globulus ssp. globulus, de 3 a 6 anos de idade, no oeste da Austrália. O maior valor de IAF observado neste trabalho foi de 4,31, em um talhão de 24 meses de idade do clone MG2, enquanto o menor valor foi de 1,72, em um talhão de 60 meses do clone MG1.

R. Árvore, Viçosa-MG, v.26, n.4, p.421-427, 2002 
Quadro 2 - Dados de IAF e idade e suas estatísticas: média ( $X_{I A F}$ ), IAF máximo e mínimo, desvio-padrão ( $S_{I A F}$ ) e correlação $(r)$

Table 2 - LAI data and age and their statistics: mean, maximum and minimum LAI, standard deviation and correlation

\begin{tabular}{|c|c|c|c|c|c|c|}
\hline Clones & IAF e Idade (meses) & $\bar{X}_{I A F}$ & $\begin{array}{c}\text { IAF } \\
\text { Máximo }\end{array}$ & $\begin{array}{c}\text { IAF } \\
\text { Mínimo }\end{array}$ & $S_{I A F}$ & $r$ \\
\hline MG1 & $\begin{array}{l}1,72(60) 2,29(56) 2,85(44) 3,08(26) 3,28(21) \\
1,82(64) 2,48(41) 2,94(18) 3,11(26) 3,41(26) \\
1,87(64) 2,61(36) 3,03(36) 3,12(26) \\
2,01(60) 2,81(40) 3,04(22) 3,14(45) \\
2,24(56) 2,84(26) 3,05(36) 3,23(26)\end{array}$ & 2,73 & 3,41 & 1,72 & 0,51 & $-0,89$ \\
\hline MG2 & $\begin{array}{l}2,16(53) 2,54(30) 2,84(68) 3,06(68) 3,47(15) \\
2,26(67) 2,64(56) 2,85(45) 3,17(40) 3,57(21) \\
2,28(70) 2,65(67) 2,88(58) 3,18(22) 3,66(26) \\
2,42(44) 2,75(76) 2,98(55) 3,21(40) 4,31(24) \\
2,48(61) 2,81(69) 3,00(32) 3,42(21)\end{array}$ & 2,94 & 4,31 & 2,16 & 0,51 & $-0,67$ \\
\hline MG3 & $\begin{array}{l}2,46(65) 2,58(65) 2,81(56) 3,24(55) \\
2,46(65) 2,80(61) 2,85(32) 3,35(26) \\
2,54(65) 2,80(61) 3,03(25) 4,02(24)\end{array}$ & 2,91 & 4,02 & 2,46 & 0,43 & $-0,76$ \\
\hline MG4 & $\begin{array}{l}2,14(35) 2,28(71) 2,52(65) 2,66(20) 3,01(65) \\
2,17(40) 2,31(70) 2,55(37) 2,81(22) 3,27(19) \\
2,17(63) 2,48(57) 2,62(19) 2,95(15)\end{array}$ & 2,57 & 3,27 & 2,14 & 0,35 & - \\
\hline MG5 & $\begin{array}{l}2,27(36) 2,70(41) 3,03(36) 3,15(78) 3,28(44) \\
2,52(66) 2,79(68) 3,03(60) 3,21(45) 3,32(68) \\
2,52(66) 2,79(68) 3,04(69) 3,24(58) 3,47(76) \\
2,66(68) 3,00(62) 3,13(62) 3,25(33) 3,54(56) \\
2,66(68) 3,01(61) 3,13(62) 3,25(59) 3,57(35)\end{array}$ & 3,04 & 3,57 & 2,27 & 0,34 & - \\
\hline Total & & 2,87 & 4,31 & 1,72 & 0,46 & $-0,77$ \\
\hline
\end{tabular}

Foi verificada correlação negativa significativa nos clones MG1, MG2 e MG3. Ares \& Fownes (2000) associaram a diminuição dos valores de IAF ao aumento de altitude local em relação ao nível do mar, no entanto tal fato não ocorreu neste estudo, uma vez que as plantações estão localizadas na região costeira, em solos de tabuleiro, de topografia basicamente plana, onde as altitudes variam de 10 a $60 \mathrm{~m}$. Os coeficientes de correlação entre IAF e idade foram mais elevados nos clones MG1 e MG3 (-0,89 e - 0,76 , respectivamente). A mesma associação foi baixa para MG2 $(-0,67)$ e não-significativa para MG4 e MG5. Estes resultados divergem daqueles de Hingston et al. (1998), que apontaram aumento de IAF com a idade. Tal diferença de comportamento, entre os estudos, pode estar

R. Árvore, Viçosa-MG, v.26, n.4, p.421-427, 2002 associada ao fato de Hingston et al. (1998) terem trabalhado com Eucalyptus globulus ssp. globulus, ao passo que neste trabalho foram estudados materiais genéticos de híbridos de Eucalyptus grandis, além de o clima e as características edáficas serem distintas nos dois estudos.

$\mathrm{Na}$ análise de regressão, a relação do IAF com a idade apresentou três comportamentos, sendo: a) uma queda não-linear do IAF com a idade (MG1 e MG3); b) uma queda linear do IAF com a idade (MG2); e c) um IAF constante ao longo do crescimento (MG4 e MG5) (Figura 1). A variação total do IAF, explicada pela idade nos modelos propostos, foi de 83,45 e $54 \%$ para os clones MG1, MG2 e MG3, respectivamente. 

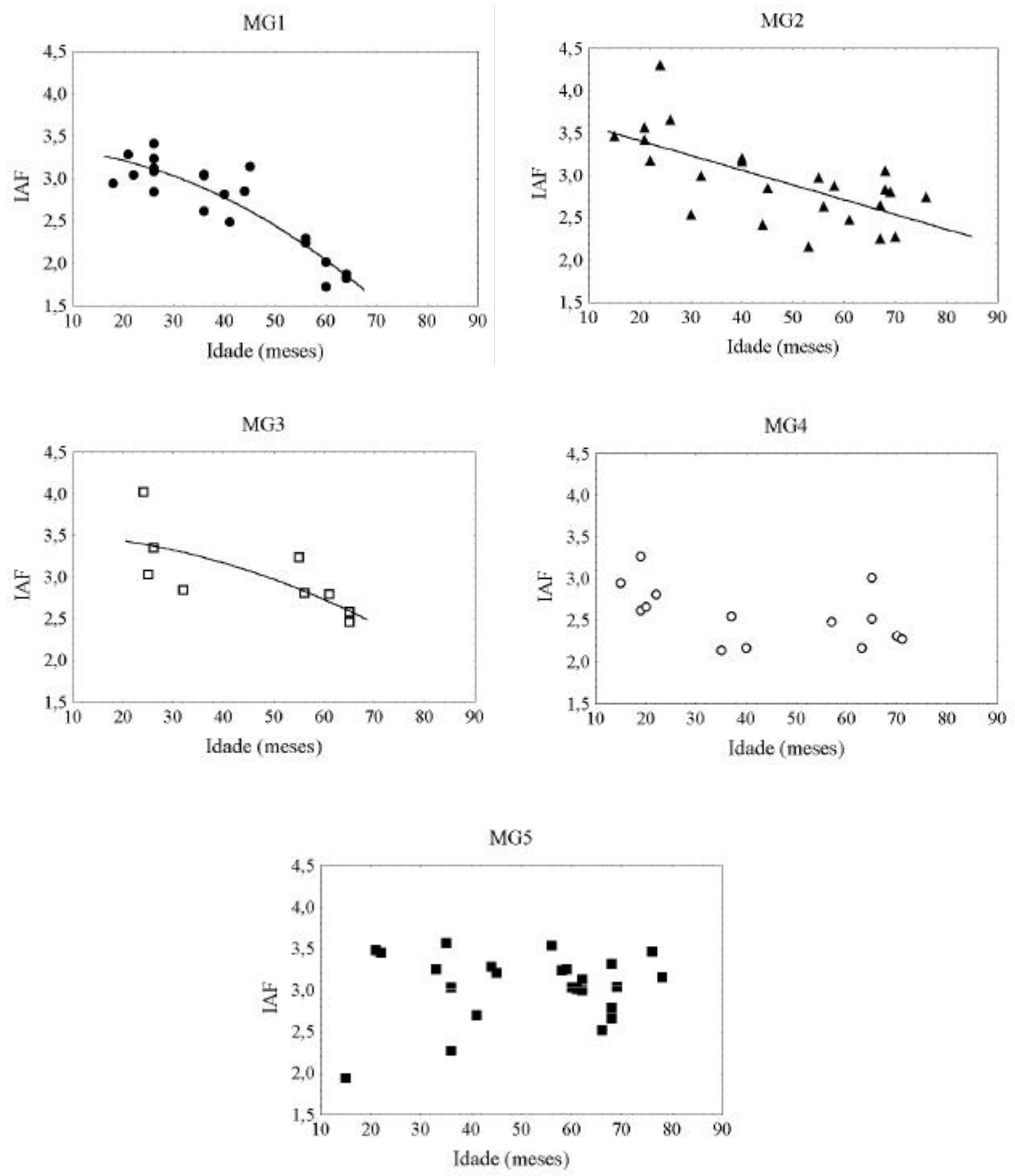

Figura 1 - Diagramas de dispersão, relacionando IAF e idade dos diferentes clones de eucalipto no norte do Espírito Santo (MG1, MG2, MG3, MG4 e MG5).

Figure 1 - LAI variation with age for different genetic materials (MG1, MG2, MG3, MG4 and MG5). 
Os modelos quadráticos, para os materiais genéticos MG1 e MG2, apresentam parâmetros distintos (nível de 95\% confiança), ou seja, os dados de IAF apresentam características de desenvolvimento diferentes ao longo do tempo.

As médias de IAF coletadas em campo, referentes a MG4 e MG5, diferiram entre si a 95\% (2,57 e 3,04, para os clones MG4 e MG5, respectivamente). Tal fato pode ser atribuído à menor média da idade entre as amostras do MG4 (42,7 meses), do que entre as de MG5 (56,4 meses)

Em trabalho realizado por Linhares et al. (2000), com plantações Pinus spp., não foi observada correlação significativa entre IAF e idade, o que pode ter ocorrido devido ao agrupamento de várias espécies diferentes na análise, devendo ser ressaltado que estas relações podem ser distintas, como evidenciam os resultados encontrados neste trabalho.

Conclui-se que as relações entre IAF e idade, apresentadas neste trabalho foram distintas para os clones analisados. O decréscimo do IAF com a idade, observado (MG1, MG2 e MG3) para os talhões de maior idade, reflete o fato de o incremento corrente anual medido regularmente pelo inventário florestal realizado nestas plantações, atingir seu pico entre três e quatro anos, decrescendo sensivelmente quando se aproxima o período de corte (sete anos). Para que o IAF dos clones MG1, MG2 e MG3 seja empregado em estudos que utilizem esta variável em modelos de processos como balanço de água usando a parametrização de Penman-Monteith, na estimativa de transpiração do dossel (Soares \& Almeida, 2001), sugere-se utilizar as relações apresentadas no Quadro 3. E, por fim, como não houve relação significativa entre IAF e idade nos clones MG4 e MG5, assumese o valor médio de IAF como sendo a melhor estimativa sobre estes para aplicação nestes modelos.

Quadro 3 - Modelos preditivos de IAF em função da idade de plantio no ciclo produtivo da plantação de eucaliptos para os clones MG1, MG2,MG3, MG4 e MG5

Table 3 - LAI models in function of age for eucalypt clone plantation (MG1, MG2, MG3, MG4 e MG5), in a production cycle

\begin{tabular}{|l|l|c|c|c|}
\hline Clone & \multicolumn{1}{|c|}{ Modelo } & R2 & Valor-p & Erro-padrão \\
\hline MG1 & IAF $=3,36100-0,0003667$ Idade $^{2}$ & 0,83 & $<0,01$ & 0,21 \\
MG2 & IAF $=3,76275-0,0174823$ Idade $^{2}$ & 0,45 & $<0,01$ & 0,39 \\
MG3 & IAF=3,52290 - 0,0002196Idade & 0,54 & $<0,01$ & 0,30 \\
MG4 & IAF $=2,75$ & - & $=0,08$ & 0,33 \\
MG5 & IAF $=3,04$ & - & $=0,37$ & 0,40 \\
\hline
\end{tabular}

R. Árvore, Viçosa-MG, v.26, n. 4, p.421-427, 2002

\section{AGRADECIMENTO}

À Aracruz Celulose S.A., pelo apoio logístico e envolvimento de técnicos durante a campanha de campo assim como a autorização para a publicação dos dados obtidos.

\section{REFERÊNCIAS BIBLIOGRÁFICAS}

ARES, A.; FOWNES, J. H. Productivity, nutrient and water-use efficiency of Eucalyptus saligna and Toona ciliata in Hawaii. Forest Ecology and Management, v. 139, p. 227-236, 2000 .

BARCLAY H. J.; TROFYMOW J. A.; LEACH R. I. Assessing bias from boles in calculating leaf area index in immature Douglas-fir with the LI-COR canopy analyzer. Agricultural and Forest Meteorology, v. 100, p. 255-260, 2000 .

BATTAGLIA, M. et al. Prediction of leaf area index in eucalypt plantations: effects of water stress and temperature. Tree Physiology, v. 18, p. 521-528, 1998.

CHEN, J. M.; CIHLAR, J. Retrieving leaf area index of boreal conifer forests using Landsat TM images. Remote Sensing of Environment, v. 55, p. 153-162, 1996.

CHEN, J. M.; BLACK, T. A.; ADAMS, R. S. Evaluation of hemispherical photography for determining plant area index and geometry of a forest stand. Agricultural and Forest Meteorology, v. 56, p. 129-143, 1991.

DAUGHTRY, C. S. T. Direct measurement of canopy structure. In: GOEL, N. S.; NORMAN, J. M. (Eds.) Instrumentation for studying vegetation canopies for remote sensing in optical and thermal infrared regions. London: Harwood, 1990. cap. 5, p. 45-60.

HINGSTON, F. J.; GALBRAITH, J. H.; DIMMOCK, G. M. Application of the process-based model BIOMASS to Eucalyptus globulus ssp. globulus plantations on exfarmland in south western Australia - I. Water use by trees and assessing risk of losses due to drought. Forest Ecology and Management, v. 106, p. 141-156, 1998.

KALLARACKAL, J.; SOMEN, C. K. An ecophysiological evaluation of the suitability of Eucalyptus grandis for planting in the tropics. Forest Ecology and Management, v. 95, p. 53-61, 1997.

LANG, A. R. G.; McMURTRIE, R. E. Total leaf areas of single trees of Eucalyptus grandis estimated from transmittances of the sun's beam. Agricultural and Forest Meteorology, v. 58, p. 79-92, 1992. 
LI-COR. LAI-2000 plant canopy analyser: Instruction manual. Li-cor Inc. Lincoln: 1992. 182 p.

LINHARES, C. A.; PONZONI, F. J.; SHIMABUKURO, Y. E. Relação entre volume de madeira e índice de área foliar em um povoamento de Pinus spp.: estudo de caso. Revista Árvore, v. 24, p. 47-54, 2000.

NORMAN, J. M.; CAMPBELL, G. S. Canopy structure. In: PEARCY, R. W.; EHLERINGER, J., MOONEY, H. A., RUNDEL, P. W. (Eds). Plant physiological ecology: field methods and instrumentation. London: Chapman and Hall, 1989. p. 301-326.

RUNNING, S. W.; COUGHLAN, J. C. A general model of forest ecosystem processes for regional applications: I. Hydrological balance, canopy gas exchange and primary production processes. Ecological Modelling, v. 42, p. 125154, 1988.

INSTITUTE. SAS/STAT User's Guide, version 6. 4.ed. Cary: 2 v.

SOARES, J. V.; ALMEIDA A. C.; PENCHEL R. M.

Balanço hídrico de plantações de eucalipto a partir da estimativa de transpiração pelo método de Penman-Monteith (Hydrological balance of eucalypt plantations through transpiration by the method of Penman-Monteith). In: INTERNATIONAL UNION OF FOREST RESEARCH ORGANIZATIONS (IUFRO) CONFERENCE ON SILVICULTURE AND IMPROVEMENT OF EUCALYPTS, 1997, Salvador. Anais... Salvador: v. 4, p. 52-61.
SOARES, J. V.; ALMEIDA, A. C. Modelling the water balance and soil water fluxes in a fast growing eucalypt plantation in Brazil. Journal of Hydrology, v. 253, p. 130147, 2001.

VERTESSY, R. A. et al. Relationships between stem diameter, sapwood area, leaf area and transpiration in a young mountain ash forest. Tree Physiology, v. 15, p. 559567, 1995.

WATSON, D. J. Comparative physiological studies on growth of field crops: I. Variation in net assimilation rate and leaf area between species and varieties, and within and between years. Annals of Botany, v. 11, p. 41-76, 1947.

WELLES, J. M. Some indirect methods of estimating canopy structure. Remote Sensing Reviews, v. 5, p. 31-34, 1990.

WELLES, J. M.; COHEN, S. Canopy structure measurement by gap fraction analysis using commercial instrumentation. Journal of Experimental Botany, v. 47, p. $1335-1347,1996$.

XAVIER, A. C. Estimativa de propriedades biofísicas de plantações de eucaliptos a partir de dados Landsat-TM. São José dos Campos, Instituto Nacional de Pesquisas Espaciais, 1998. 86 p. Dissertação (Mestrado em Sensoriamento Remoto) - Instituto Nacional de Pesquisas Espaciais, 1998. 